\title{
¿Quiénes son los sentenciados por homicidio intencional en México?
}

\section{Carlos Vilalta*}

\author{
Perfiles Latinoamericanos | Artículo \\ vol. 23 | núm. 45 | 2015 \\ pp. $157-180$
}

\begin{abstract}
Resumen
Se presenta un perfil preliminar del homicida intencional en México. De esta forma se dan los primeros pasos empíricos en la construcción de un conocimiento sistemático y comparativo del delincuente violento. Se comparó al total de condenados por homicidio intencional frente al total de condenados por otros delitos federales intencionales entre 1997 y 2010 . Se encontró que el total de sentenciados por este delito en el fuero federal es muy reducido, su número varía mucho entre ańos y cada vez es proporcionalmente menor frente a los sentenciados por otros delitos intencionales. También se encontró que cerca de un tercio cometió su delito fuera del país, principalmente en los Estados Unidos. Finalmente, se encontraron proporciones significativamente mayores de sentenciados casados, con grados universitarios y ocupados como profesionistas entre los homicidas intencionales.
\end{abstract}

\begin{abstract}
We present a preliminary profile of the murderer in Mexico. This is the first step in constructing an empirical knowledge and systematic base for the comparative study of violent offenders in the Latin American region. The total number of federal convicts for murder was compared with the total number of convicts for other intentional crimes between 1997 and 2010. The study found that the total number of federal convicts for murder is comparatively small, highly varying and becoming less over time. About one third committed their crime overseas, mostly in the United States. Also, significantly higher proportions of married, college degrees, and professionals were found among convicts for murder.
\end{abstract}

Palabras clave: homicidio, delito, estadística delictiva, México.

Keywords: homicide, crime, crime statistics, Mexico.

* Doctor en Estudios Urbanos por la Universidad Estatal de Portland (Estados Unidos) y profesor investigador titular de la División de Estudios Jurídicos del CIDE. 


\section{Introducción}

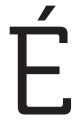

sta es una investigación sobre delincuentes violentos y se centra en los sentenciados por el delito de homicidio doloso o intencional del fuero federal en México entre 1997 y 2010. Los motivos de concentrarse en la población de homicidas intencionales en fuero federal son dos: primero, la prácticamente nula información existente al respecto de este fuero en oposición al fuero común, ${ }^{1} \mathrm{y}$, segundo, por el proceso actual de modernización del sistema penitenciario federal, su reordenación territorial, y por las nuevas políticas de reinserción social de este segmento de la población carcelaria en el país. ${ }^{2}$ Sólo por dar un ejemplo del reciente y enorme proceso de cambio del sistema penitenciario federal, si en 2006 el país contaba con 44000 reclusos por delitos federales y solamente el $7.2 \%$ estaba bajo custodia del gobierno federal (el resto se hallaba distribuido en prisiones estatales y entremezclado con los reclusos por delitos del fuero común), para 2012 ya se contaba con cerca de 50000 reclusos en este ámbito y con 21 prisiones federales.

El estudio de los homicidas dolosos en el fuero federal es indispensable por un motivo criminológico simple: su alta peligrosidad medida no sólo por el tipo de delito cometido per se sino por su experiencia en el uso de armas de fuego y su alta tasa de reincidencia. En una encuesta reciente aplicada a una muestra representativa de reclusos en cárceles federales de México, ${ }^{3}$ se encontró que el $12.9 \%$ de los reclusos por homicidio doloso reportó haber prestado sus servicios en la policía o el ejército, y la misma proporción, el 12.9\%, reportó también haber estado en la cárcel anteriormente por otro delito (principalmente robo simple y portación de arma prohibida). También cabe mencionar que el 41.9\% de este grupo de sentenciados por homicidio doloso reportó haber estado en los Estados Unidos y que de éstos, casi una tercera parte (30.8\%), también estuvo detenido en ese país (principalmente por su situación migratoria, por cometer un robo, o por conducir ebrio, en ese orden de frecuencia).

Es así que este documento forma parte de un estudio comparativo y de largo plazo sobre perfiles criminales en el continente americano. El enfoque es principalmente estadístico y se fundamenta en información oficial y pública. En este sentido, no se conoce de un estudio similar que haga uso de estadísticas oficiales para la región, pese a la importancia en la agenda pública actual que

\footnotetext{
1 Sobre reclusos en el fuero común se cuenta con los estudios de Vilalta y Fondevila (2013).

2 Cabe agregar que la población carcelaria en el país se triplicó entre 1994 y 2011, pasando de 86 mil a 231 mil, respectivamente.

3 Primera Encuesta a Población Interna en Centros Federales de Readaptación Social, C. Pérez et al. (2012).
} 
poseen las áreas de seguridad y justicia. El objetivo central de este estudio, en lo particular, es el de llegar a una delimitación básica de las características propias de los homicidas intencionales en comparación con los sentenciados por otros delitos. Los propósitos que dieron origen a esta investigación fueron dos: ofrecer información actual y detallada sobre un problema social poco estudiado empíricamente en la región, y motivar la realización de estudios similares comparativos en la región latinoamericana, elevando así la calidad de la información estadística criminal en la región.

El análisis estadístico se desarrolla sobre nueve correlativos disponibles en las estadísticas judiciales penales del Instituto Nacional de Estadística y Geografía de México (INEGI). Estos son: lugar de ocurrencia, género, edad, nacionalidad, situación conyugal, escolaridad, ocupación, estado psicofísico, y grado de consumación. El análisis muestra frecuencias, estadísticos descriptivos y pruebas de diferencias entre grupos. En este caso, se realizan inferencias sobre comparaciones entre sentenciados por homicidio doloso y la población general de sentenciados por otros delitos dolosos o intencionales. Es decir, ¿qué características podrían llegar a distinguir a cada grupo de reclusos, siendo el dolo o la intención el factor común a cada uno?

A continuación, el documento se divide en tres secciones. Se inicia con la descripción detallada de la información y datos utilizados. La segunda sección muestra el análisis de los correlativos de los homicidas dolosos entre 1997 y 2010. La tercera, es una discusión sobre los hallazgos y algunas ideas sobre lo que sigue estudiar.

\section{Información y metodología}

Es importante considerar que, al igual que el comportamiento del homicida intencional debe ser visto como un proceso, ${ }^{4}$ las estadísticas oficiales sobre la materia reflejan un proceso, en este caso, administrativo y judicial (gráfico 1). ${ }^{5}$ Más a detalle, las mediciones se realizan sobre tres tipos diferentes, pero interconectadas de actividad gubernamental: actividad policial, judicial, y penitenciaria. Estas actividades pueden también verse en las tres partes según el tipo de organismo público que actúa y se hace cargo de la etapa en el proceso, y que a su vez produce sus propias estadísticas de volumen y tipo de actividad:

4 Con correlativos demográficos, de personalidad, de contexto familiar y comunitarios identificables, mensurables, y que pueden ser considerados como causas conducentes de tal comportamiento.

5 Presupuestalmente, y aunque incluye a muchas otras instituciones (por ej., la Comisión Nacional de Derechos Humanos), en el Presupuesto de Egresos de la Federación a esta función se le denomina "Orden, Seguridad y Justicia”. 
estadísticas de seguridad pública y procuración de justicia, estadísticas judiciales y estadísticas penitenciarias (gráfico 1). Como en todas las áreas de gobierno, las instituciones de seguridad y justicia se hacen cargo y miden aspectos diferentes de la criminalidad. Este estudio, y particularmente esta sección, se desarrolla sobre la base de información de la segunda etapa o proceso de medición y que es la actividad judicial, en dos grupos de población: procesados y sentenciados. "Procesado" o "presunto delincuente" es el término dado a las personas a quienes el juez de primera instancia les ha dictado Auto de Término Constitucional relativo al/los delito(s) por el/los que fueron consignados (INEGI, 2007). ${ }^{6}$ A su vez, "sentenciado" es el término estadístico que se refiere a la persona a quien el juez de primera instancia le ha dictado sentencia, ya sea absolutoria o condenatoria (INEGI, 2007).

Gráfico 1. México: el proceso de medición de la actividad delictiva*

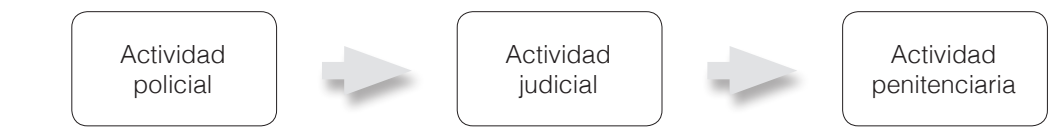

* Este estudio se fundamenta en información proveniente de la actividad judicial.

Fuente: Elaboración propia.

Enseguida, este estudio se centra en examinar estadísticamente la significancia de diez correlativos de diferentes tipos entre los sentenciados por homicidio doloso (cuadro 1). Los correlativos examinados son: lugar de residencia, lugar de ocurrencia del delito, género, edad, nacionalidad, situación conyugal, escolaridad, ocupación, estado psicofísico, y grado de consumación.

Esto se realiza de manera comparativa con el general de los sentenciados por delitos federales dolosos y en una perspectiva histórica hasta donde lo permite la disponibilidad de información estadística judicial penal (1997 a 2010). Es importante aclarar que la comparación se realiza frente al total (general) de la población bajo estudio, es decir, todos aquellos que cometieron algún delito doloso, incluyendo los procesados por homicidio doloso. ${ }^{7} \mathrm{Al}$ final de la sec-

6 Véase la Sintesis metodológica de las estadísticas judiciales penales, disponible en <http://goo.gl/ipl92y>.

7 Esto se hizo por limitaciones de tiempo, pero es infinitamente poco probable que los resultados de las pruebas inferenciales resulten afectadas visto que los homicidas dolosos representan una apenas perceptible minoría del total de procesados por algún delito con intencionalidad dolosa en el fuero federal. 


\section{ción correspondiente, se muestra la síntesis de cuáles fueron los correlativos o características propias que desde este acercamiento estadístico inicial resultaron asociados a los sentenciados por homicidio doloso en el fuero federal.}

Cuadro 1. Variables para la delimitación del perfil

\begin{tabular}{|c|c|}
\hline Concepto & Medición \\
\hline Lugar de residencia & Entidad federativa \\
\hline Lugar de ocurrencia del delito & Entidad federativa \\
\hline Género & $\begin{array}{l}\text { Hombre } \\
\text { Mujer }\end{array}$ \\
\hline Grupos de edad & $\begin{array}{l}18 \text { a } 19 \text { años } \\
20 \text { a } 24 \text { años } \\
25 \text { a } 29 \text { años } \\
30 \text { a } 34 \text { años } \\
35 \text { a } 39 \text { años } \\
40 \text { a } 44 \text { años } \\
45 \text { a } 49 \text { años } \\
50 \text { a } 54 \text { años } \\
55 \text { a } 59 \text { años } \\
60 \text { y más años }\end{array}$ \\
\hline Nacionalidad & $\begin{array}{l}\text { Mexicana } \\
\text { Extranjera }\end{array}$ \\
\hline Situación conyugal & $\begin{array}{l}\text { Soltero } \\
\text { Casado } \\
\text { Viudo } \\
\text { Divorciado } \\
\text { Unión libre } \\
\text { Separado }\end{array}$ \\
\hline Escolaridad & $\begin{array}{l}\text { Primaria } \\
\text { Secundaria } \\
\text { Preparatoria } \\
\text { Profesional }\end{array}$ \\
\hline \multirow[t]{2}{*}{ Ocupación } & $\begin{array}{l}\text { Población económicamente activa (PEA): } \\
\text { Profesionistas } \\
\text { Técnicos } \\
\text { Trabajadores de la educación } \\
\text { Trabajadores del arte } \\
\text { Funcionarios y directivos } \\
\text { Trabajadores agropecuarios } \\
\text { Inspectores y supervisores } \\
\text { Artesanos y obreros } \\
\text { Operadores de maquinaria fija } \\
\text { Ayudantes y similares } \\
\text { Operadores de transporte } \\
\text { Coords. y supervisores admvos. } \\
\text { Oficinistas } \\
\text { Comerciantes y dependientes } \\
\text { Trabs. ambulantes } \\
\text { Trabs. en servicios públicos } \\
\text { Trabs. domésticos } \\
\text { Protección y vigilancia }\end{array}$ \\
\hline & Población no económicamente activa (NO PEA) \\
\hline Estado psicofísico & $\begin{array}{l}\text { En pleno uso de sus facultades } \\
\text { Ebrio } \\
\text { Drogado } \\
\text { Otro estado } \\
\text { Se ignora }\end{array}$ \\
\hline Grado de consumación & $\begin{array}{l}\text { Tentativa } \\
\text { Consumado }\end{array}$ \\
\hline
\end{tabular}

Fuente: Elaboración propia sobre la información del INEGI. 
La información disponible en las estadísticas judiciales mexicanas no es tan detallada como se desearía. No obstante, existen algunas ventajas iniciales, y pueden observarse sobre la base de las variables revisadas para la delimitación del perfil en relación con grandes grupos de correlativos (gráfico 2). Esta clasificación de grandes grupos de correlativos tiene inspiración en el trabajo previo de Ellis et al. (2009).

En tal empalme, quedaría por clasificar el grado de consumación del delito, el cual puede depender de una amplia variedad de circunstancias fuera del control del victimario. En este caso, probablemente la categoría de mayor interés analítico sería la tentativa. La tentativa se define como la ejecución incompleta de las acciones encaminadas, directa e inmediatamente, a cometer un delito, el cual no se consumó por causas ajenas a la voluntad del victimario (INEGI, 2007). Es decir, no se trata de un desistimiento sino de un fallo en la ejecución del delito. ${ }^{8}$ La tentativa es analíticamente interesante porque da indicios de la determinación, la capacidad individual y la dificultad de cometer un delito. En este caso, la comparación proporcional de tentativas por tipos diferentes de delitos (por ej., homicidio doloso y otros actos dolosos) permite introducir matices en tales aspectos de determinación y capacidad de los delincuentes y la dificultad en la comisión de los delitos.

Gráfico 2. Grupos de correlativos en la literatura científica y variables disponibles en las estadísticas judiciales penales de México

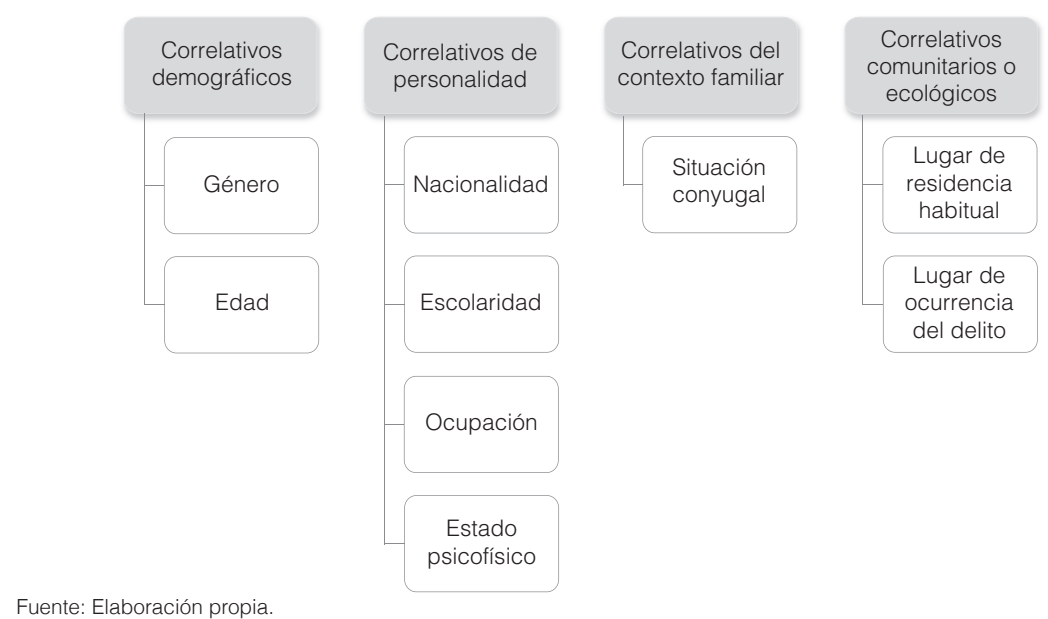

8 Si el criminal no actúa con la voluntad consciente de cometer un acto delictivo (dolo), no puede haber tentativa. 
Naturalmente, es debatible si la nacionalidad, la escolaridad y la ocupación proveen de una "personalidad" al victimario. La personalidad son las diferencias individuales que constituyen a una persona y que la distinguen de otra, o dicho de otra manera, se trata del conjunto de características o cualidades originales que destacan en algunas personas (RAE, 2011). En este sentido, se podría argumentar de manera muy elemental que tales variables (1) sí pueden producir características o cualidades originales o mayoritarias en ciertos grupos de individuos, por ejemplo, sobre patrones conductuales o expectativas de vida que, a su vez, pueden (2) generar comportamientos, identidades y reacciones diferentes en situaciones similares. El estado psicofísico sí se puede considerar de manera relativamente directa como un correlativo de la personalidad, puesto que el estado de intoxicación reduce los niveles de autocontrol del individuo y modifica tales comportamientos y reacciones (Vilalta, 2010; Shaw et al., 2006; Leri et al., 2003; Makkai, y Payne, 2003).

Los desempalmes y limitaciones de información previamente mencionados tuvieron el efecto natural de limitar la capacidad de análisis del estudio. Sin embargo, considerando las pretensiones introductorias de éste y la buena calidad de la información, así como las evidencias que pudieron ser extraídas del examen estadístico de los datos judiciales penales nacionales, todo esto, en su conjunto, permitió delimitar un perfil del homicida doloso sobre bases científicamente compartidas de información que cubren estándares de evidencia científica.

Acerca de tales correlativos, el perfil se delimita comparando sistemáticamente entre dos grupos de sentenciados: aquellos por homicidio doloso y la población general de sentenciados por otros delitos dolosos o intencionales. Las inferencias en las comparaciones fueron hechas a través de pruebas estándar de diferencias de proporciones. ${ }^{?}$

\section{Correlativos}

Lo primero que se observa en términos de sentenciados por homicidio doloso, y en general en el fuero federal, son dos cosas: el incremento y la mayor participación de sentenciados por ese delito dentro del total. ${ }^{10}$ Los sentenciados entre 1997 y 2010 por delitos dolosos en el fuero federal sumaron 375036

9 Véase el anexo estadístico al finalizar el artículo.

10 Aplica la misma advertencia sobre los totales en cada cuadro en relación con la no inclusión de los No Especificados (NE) en la base de datos original del INEGI. 
personas; casi 27000 individuos por año. ${ }^{11}$ En el periodo, la proporción de sentenciados por homicidio doloso, del total de sentenciados por algún delito doloso, es muy baja: $0.14 \%$. Sólo se ha sentenciado a 533 personas por este delito. También se observa que tal proporción es sumamente variable entre años, ya que oscila entre $0.08 \%$ y $0.25 \%$.

Cuadro 2. Homicidio y general (dolosos): número y proporción de sentenciados, de 1997 a 2010

\begin{tabular}{|c|c|c|c|c|c|}
\hline & \multicolumn{2}{|c|}{ Homicidio dolosos } & \multicolumn{2}{|c|}{ General (dolosos) } & \multirow{2}{*}{$\begin{array}{c}\text { Proporción de sentenciados por } \\
\text { homicidio doloso (en \%)* }\end{array}$} \\
\hline & Doloso & Otro* & Doloso & Otro* $^{*}$ & \\
\hline 1997 & 25 & 76 & 19887 & 4837 & 0.13 \\
\hline 1998 & 47 & 92 & 21575 & 4067 & 0.22 \\
\hline 1999 & 28 & 45 & 24614 & 349 & 0.11 \\
\hline 2000 & 21 & 32 & 23376 & 168 & 0.09 \\
\hline 2001 & 23 & 25 & 24611 & 131 & 0.09 \\
\hline 2002 & 37 & 31 & 26351 & 150 & 0.14 \\
\hline 2003 & 45 & 38 & 27599 & 197 & 0.16 \\
\hline 2004 & 39 & 18 & 28806 & 134 & 0.14 \\
\hline 2005 & 44 & 33 & 28550 & 144 & 0.15 \\
\hline 2006 & 73 & 25 & 29182 & 118 & 0.25 \\
\hline 2007 & 47 & 22 & 28379 & 85 & 0.17 \\
\hline 2008 & 32 & 14 & 27932 & 78 & 0.11 \\
\hline 2009 & 43 & 26 & 28314 & 386 & 0.15 \\
\hline 2010 & 29 & 20 & 35860 & 364 & 0.08 \\
\hline Total & 533 & 497 & 375036 & 11208 & 0.14 \\
\hline
\end{tabular}

*Del total de sentenciados por delitos dolosos.

Fuente: Elaboración propia con base en las Estadísticas judiciales en materia penal (INEGI). Los datos se refieren al número de sentenciados por año de registro.

Pasando a la observación del lugar de residencia de los sentenciados por homicidio doloso, encontramos una minoría que reside fuera del país (1.7\%), la cual no parece ser estadísticamente diferente a la del general de los sentenciados en este fuero por algún delito doloso (1.4\%). Cabe mencionar que, de manera similar a los procesados, la mayor parte de sentenciados que residían fuera del país, se encontraban en los Estados Unidos (8 o $1.5 \%$ del total). ${ }^{12}$ $\mathrm{Al}$ interior del país, las tres entidades con los mayores números de residentes sentenciados por homicidio doloso en el periodo 1997-2010 han sido el Distrito Federal $(\mathrm{N}=58)$, Jalisco $(\mathrm{N}=56)$ y el Estado de México $(\mathrm{N}=48)$.

11 Los sentenciados pueden serlo por uno o más delitos.

12 Véase el cuadro respectivo en el anexo estadístico al concluir este artículo. 
Cuadro 3. Homicidio y general (dolosos): número y proporción de sentenciados por lugar de residencia habitual, 1997 a 2010*

\begin{tabular}{l|cc|cc}
\cline { 2 - 4 } & \multicolumn{2}{|c|}{ Homicidio doloso } & \multicolumn{2}{c}{ General (dolosos) } \\
\cline { 2 - 4 } & $N$ & $\%$ & 368639 & $\%$ \\
\hline Interior del país & 522 & 98.3 & 5262 & 98.6 \\
Fuera del país** $^{*}$ & 9 & 1.7 & 1.4 \\
\hline
\end{tabular}

${ }^{*}$ No se incluyeron los sentenciados con lugar de residencia habitual no especificada (NE).

**Se refiere a Estados Unidos de América, Latinoamérica y otros países.

Fuente: Elaboración propia con base en las Estadísticas judiciales en materia penal (INEGI).

Cuadro 4. Homicidio y general (dolosos): número y proporción de sentenciados por lugar de ocurrencia del delito, 1997-2010*

\begin{tabular}{l|cc|cc}
\cline { 2 - 4 } & \multicolumn{2}{|c|}{ Homicidio doloso } & \multicolumn{2}{c}{ General (dolosos) } \\
\cline { 2 - 4 } & $N$ & $\%$ & 374089 & $\%$ \\
\hline Interior del país & 349 & 68.7 & 313 & 0.92 \\
Fuera del país** & 159 & 31.3 &
\end{tabular}

* No se ineluyeron los senteneiados con lugar de oeurreneia no especifieada (NE).

**Se refiere a Estados Unidos de América, Latinoamérica y otros países.

Fuente: Elaboración propia con base en las Estadísticas judiciales en materia penal (INEGI).

Mapa 1. Homicidio doloso: sentenciados en el fuero federal por lugar de ocurrencia del delito, 1997-2010*

Sentenciados por un lugar de ocurrencia

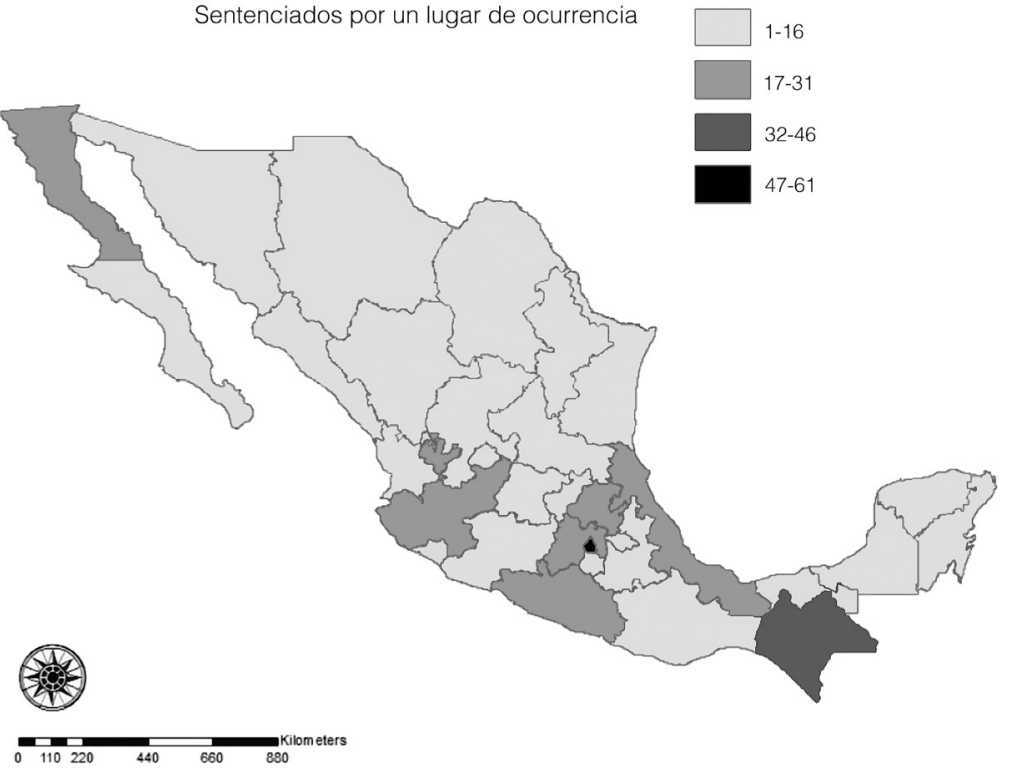

*No se incluyeron los sentenciados con lugar de ocurrencia no especificada (NE).

Fuente: Elaboración propia con base en las Estadísticas judiciales en materia penal (INEGI).

Perfiles Latinoamericanos | vol. 23, núm. 45, enero-junio de 2015 | FLACso México 
Si bien la mayor proporción de los sentenciados cometieron el homicidio doloso en algún estado del país, llama notablemente la atención que cerca de un tercio lo cometió fuera (31.3\%), es decir, tuvo que ser extraditado para ser procesado y, eventualmente, sentenciado en México. ${ }^{13}$ En el mismo periodo (1997-2010), las tres entidades federativas con las mayores proporciones de homicidas dolosos sentenciados en el fuero federal fueron el Distrito Federal $(12.0 \%)$, Chiapas $(6.7 \%)$ y Jalisco $(4.5 \%)$.

En términos del género del sentenciado, igualmente se puede esperar que sea de sexo masculino (92.3\%), no obstante, la expectativa se cumple con variaciones proporcionales relativamente notables por año y, en ocasiones, por grupo general de sentenciados por algún delito doloso; por ejemplo, en los años de 1998, 2001 y 2006 ( $\mathrm{p}<0.000)$. Sin embargo, no se observa para el periodo completo una mayor proporción de hombres (o mujeres) sentenciados por este delito, en comparación con el general de los sentenciados por algún delito doloso $(\mathrm{p}=0.782)$.

Cuadro 5. Homicidio y general (dolosos): proporción de hombres sentenciados, 1997-2010

\begin{tabular}{|c|c|c|c|c|c|c|}
\hline & \multicolumn{2}{|c|}{ Homicidio doloso } & \multicolumn{2}{|c|}{ General (dolosos) } & \multicolumn{2}{|c|}{ Prueba de diferencias* } \\
\hline & $N$ & Hombres \% & $N$ & Hombres \% & $Z$ & Sig. \\
\hline 1997 & 25 & 84.0 & 19887 & 94.6 & -1.32 & 0.187 \\
\hline 1998 & 47 & 100.0 & 21575 & 94.7 & 33.75 & 0.000 \\
\hline 1999 & 28 & 96.4 & 24614 & 94.0 & 0.67 & 0.503 \\
\hline 2000 & 21 & 95.2 & 23376 & 92.8 & 0.52 & 0.606 \\
\hline 2001 & 23 & 100.0 & 24611 & 93.0 & 41.52 & 0.000 \\
\hline 2002 & 37 & 97.3 & 26351 & 93.0 & 1.59 & 0.111 \\
\hline 2003 & 45 & 88.9 & 27599 & 92.6 & -0.74 & 0.461 \\
\hline 2004 & 39 & 76.9 & 28806 & 91.9 & -1.95 & 0.051 \\
\hline 2005 & 44 & 79.5 & 28550 & 92.1 & -1.84 & 0.066 \\
\hline 2006 & 73 & 100.0 & 29182 & 91.4 & 50.08 & 0.000 \\
\hline 2007 & 47 & 95.7 & 28379 & 91.4 & 1.43 & 0.153 \\
\hline 2008 & 32 & 87.5 & 27932 & 91.9 & -0.71 & 0.479 \\
\hline 2009 & 43 & 93.0 & 28314 & 92.4 & 0.17 & 0.867 \\
\hline 2010 & 29 & 93.1 & 35860 & 92.6 & 0.11 & 0.915 \\
\hline Total & 533 & 92.3 & 347433 & 92.6 & -0.28 & 0.782 \\
\hline
\end{tabular}

*Una significancia menor a 0.05 indicaría una diferencia estadísticamente relevante entre los porcentajes de hombres sentenciados por homicidio y hombres sentenciados en general por delitos dolosos.

Fuente: Elaboración propia con base en las Estadísticas judiciales en materia penal (INEGI).

13 Principalmente de los Estados Unidos. Véanse los cuadros en el anexo estadístico al final de este texto. 
Para los sentenciados también se calculó y graficó el índice de masculinidad (IMA) de este delito y el general de los dolosos. Recordemos que el IMA nos indica cuántos hombres por mujer fueron sentenciados y, en este caso, por año. Entre los sentenciados por homicidio doloso, la variación anual es muy notable, lo que relativamente complica la proyección hacia el futuro de la variable por género. También destaca que, de manera correlacionada con los procesados, el IMA ha venido reduciéndose para los sentenciados por homicidio doloso, lo que sugiere una posible mayor participación de mujeres en este tipo de delito en el fuero federal.

Gráfico 3. Homicidio y general (dolosos): Índice de Masculinidad (IMA) entre sentenciados, de 1997 a 2010*

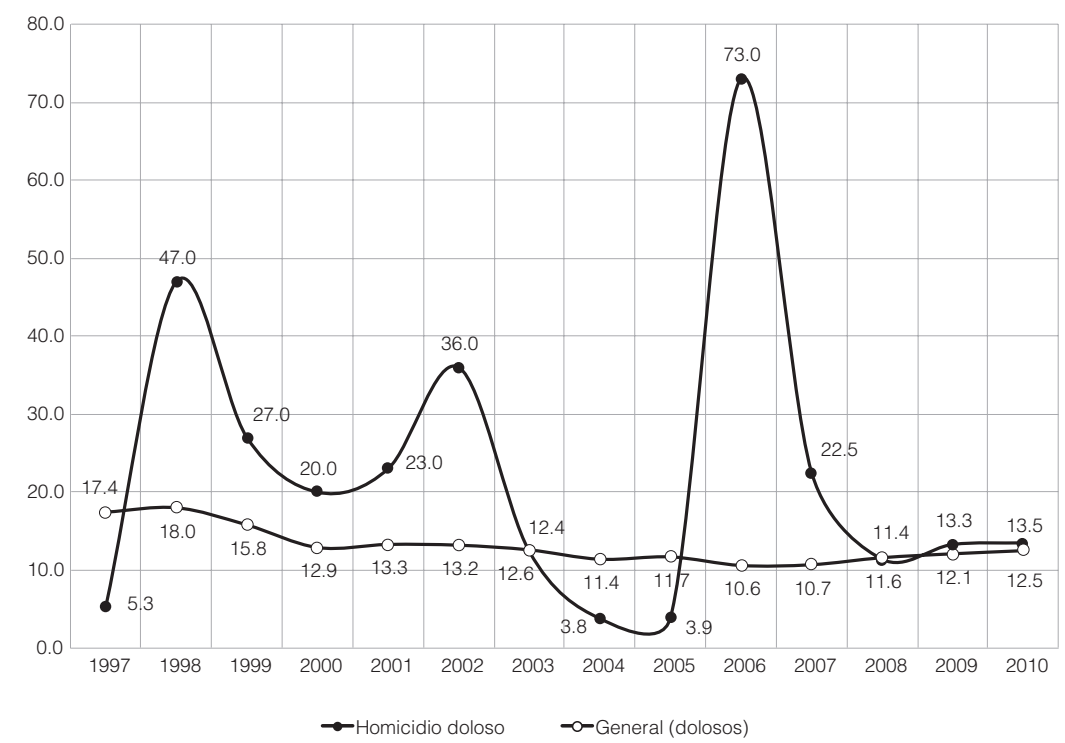

*El Índice de Masculinidad indica el número de hombres por mujer.

Fuente: Elaboración propia con base en las Estadísticas judiciales en materia penal (INEGI).

Sobre la edad de los sentenciados, se observan proporciones elevadas de homicidas dolosos en los grupos de edad de 30 a 34 años (20.5\%) y 20 a 25 años (15.9\%). En cambio, entre los sentenciados por algún delito doloso o grupo general de los sentenciados en este fuero, se observan las mayores frecuencias en los 25 a 29 años y 20 a 24 años. 
Gráfico 4. Homicidio y general (doloso): pirámide de edades, 1997-2010*

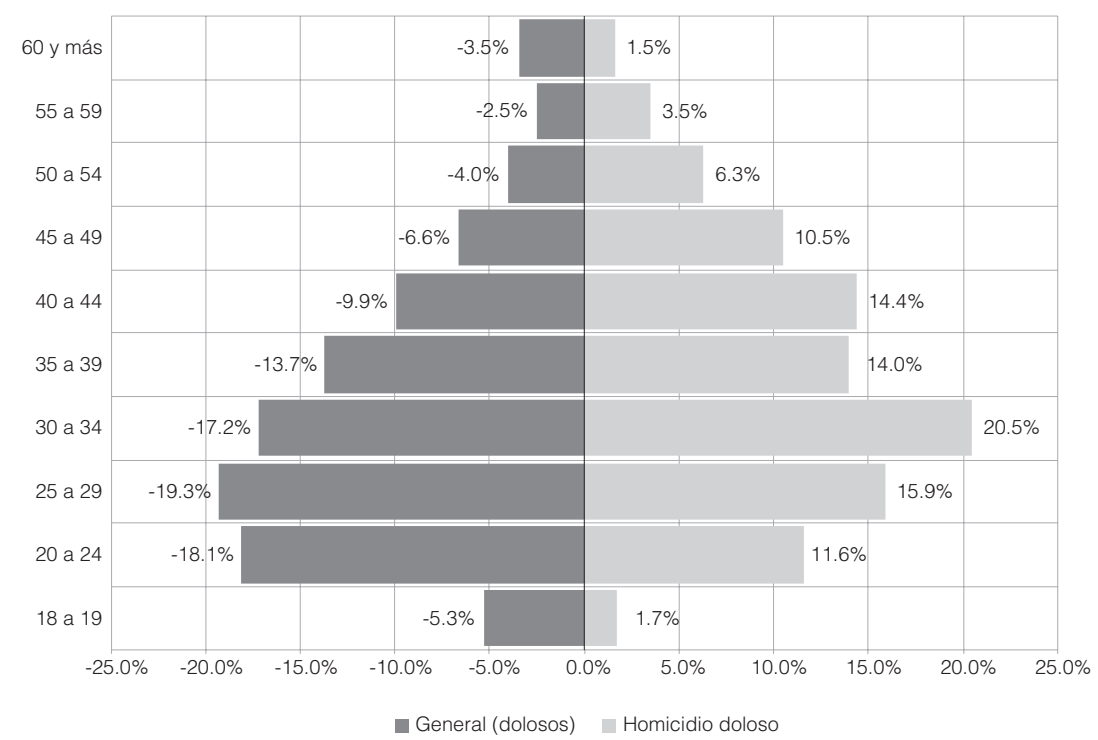

*No se incluyeron los sentenciados con edad no especificada (NE).

Fuente: Elaboración propia con base en las Estadísticas judiciales en materia penal (INEGI).

No obstante, en términos probabilísticos, no se observan diferencias estadísticamente significativas entre los sentenciados por homicidio doloso $\mathrm{u}$ otros delitos dolosos en términos de grupos de edad, al menos en el periodo 1997-2010 (véase el cuadro 6). ${ }^{14}$ Es decir, si bien entre los procesados encontrábamos diferencias significativas por grupos de edades, particularmente una mayor frecuencia de procesados por homicidio doloso con edades relativamente mayores que en el grupo general de los procesados por algún delito doloso, en este caso, sobre los sentenciados no esperaríamos, al menos con base en la evidencia presente, una mayor probabilidad de encontrar más o menos sentenciados por este delito de homicidio doloso por grupo de edad que entre la generalidad de los sentenciados.

14 Es importante recordar que los resultados de las pruebas de significancia dependen de los tamaños de las muestras; a mayores tamaños de muestras corresponden mayores probabilidades de encontrar diferencias estadísticamente significativas. En este caso, los tamaños de muestra por grupos de edades al interior de los sentenciados por homicidio doloso en varias categorías es de un tamaño muy reducido (por ej., 8, 16 y 7). Se podrían realizar pruebas de significancia con correcciones por tamańo de muestra (por ejemplo, ajustando los valores de $\mathrm{Z}$ a una distribución t de Student), pero dadas las limitaciones de tiempo esto no se pudo realizar. En todo caso, a juicio del autor, es poco probable que los resultados variaran como consecuencia de la corrección, al menos en esta ocasión y dados los excesivamente reducidos tamaños de muestra en cada categoría. 
Cuadro 6. Homicidio y general (dolosos): sentenciados por grupos de edad, 1997-2010

\begin{tabular}{c|cc|cc|cc}
\hline \multirow{2}{*}{$\begin{array}{c}\text { Grupo de edad } \\
\text { (en años) }\end{array}$} & \multicolumn{2}{|c|}{ Homicidio doloso } & \multicolumn{2}{c|}{ General (dolosos) } & \multicolumn{2}{c}{ Prueba de diferencias* } \\
\cline { 2 - 6 } & $N$ & $\%$ & $N$ & $\%$ & $Z$ & Sig. \\
\hline $18-19$ & 8 & 1.7 & 16421 & 5.3 & -0.77 & 0.442 \\
$20-24$ & 53 & 11.6 & 56027 & 18.1 & -1.49 & 0.136 \\
$25-29$ & 73 & 15.9 & 59547 & 19.3 & -0.77 & 0.439 \\
$30-34$ & 94 & 20.5 & 53063 & 17.2 & 0.81 & 0.420 \\
$35-39$ & 64 & 14.0 & 42283 & 13.7 & 0.07 & 0.945 \\
$40-44$ & 66 & 14.4 & 30460 & 9.9 & 1.05 & 0.292 \\
$45-49$ & 48 & 10.5 & 20489 & 6.6 & 0.87 & 0.384 \\
$50-54$ & 29 & 6.3 & 12401 & 4.0 & 0.51 & 0.608 \\
$55-59$ & 16 & 3.5 & 7768 & 2.5 & 0.21 & 0.831 \\
60 y más & 7 & 1.5 & 10737 & 3.5 & -0.42 & 0.675 \\
\hline
\end{tabular}

* Una significancia menor a 0.05 indicaría una diferencia estadísticamente relevante entre los porcentajes de sentenciados por homicidio y sentenciados en general por delitos dolosos. No se incluyeron los procesados con edad no especificada (NE).

Fuente: Elaboración propia con base en las Estadísticas judiciales en materia penal (INEGI).

Siguiendo con la variable de nacionalidad, también de manera correlacionada con la población de procesados, los sentenciados por homicidio doloso de nacionalidad extranjera son claramente una minoría (1.9\%). Tampoco parece ser significativamente diferente del resto de los sentenciados por un delito doloso.

Cuadro 7. Homicidio y general (dolosos): sentenciados por nacionalidad, 1997-2010*

\begin{tabular}{l|cc|cc|cc}
\cline { 2 - 6 } & \multicolumn{2}{c|}{ Homicidio doloso } & \multicolumn{2}{c|}{ General (dolosos) } & \multicolumn{2}{c}{ Prueba de diferencias* } \\
\cline { 2 - 6 } & $N$ & $\%$ & $N$ & $\%$ & $Z$ & Sig. \\
\hline Mexicana & 523 & 98.1 & 369564 & 98.8 & -1.07 & 0.286 \\
Extranjera & 10 & 1.9 & 4649 & 1.2 & 0.15 & 0.883 \\
\hline
\end{tabular}

* Una significancia menor a 0.05 indicaría una diferencia estadísticamente relevante entre los porcentajes de sentenciados por homicidio y sentenciados en general por delitos dolosos. Se incluyen los resultados para ambas proporciones de la variable dicotómica, en donde la significancia es diferente a razón de diferentes errores estándar, los cuales son explicados por los tamaños divergentes de muestra en cada categoría. No se incluyeron los sentenciados con nacionalidad no especificada (NE).

Fuente: Elaboración propia con base en las Estadísticas judiciales en materia penal (INEGI).

Sobre la situación conyugal, se observa entre los sentenciados por homicidio doloso que con frecuencia, o probablemente, están casados (53.0\%), proporción que, además, está significativamente por encima del conjunto general de sentenciados por delitos dolosos en el fuero federal $(43.9 .0 \%$ y $\mathrm{p}=0.002)$. Le siguen los solteros (24.8\%) y también se observa que alrededor de uno de cada cinco sentenciados vivía en unión libre.

Cuadro 8. Homicidio y general (dolosos): sentenciados por situación conyugal, 1997-2010

Perfiles Latinoamericanos | vol. 23, núm. 45, enero-junio de 2015 | Flacso México Carlos Vilalta | ¿Quiénes son los sentenciados por homicidio intencional en México? | pp. 157-180 


\begin{tabular}{l|cc|cc|cc} 
& \multicolumn{2}{|c|}{ Homicidio doloso } & \multicolumn{2}{c|}{ General (dolosos) } & \multicolumn{2}{c}{ Prueba de diferencias* $^{*}$} \\
\cline { 2 - 7 } & $N$ & $\%$ & $N$ & $\%$ & $Z$ & Sig. \\
\hline Soltero & 131 & 24.8 & 117672 & 31.8 & -1.85 & 0.064 \\
Casado & 280 & 53.0 & 162599 & 43.9 & 3.04 & 0.002 \\
Viudo & 8 & 1.5 & 3891 & 1.1 & 0.11 & 0.915 \\
Divorciado & 7 & 1.3 & 5621 & 1.5 & -0.04 & 0.964 \\
Unión libre & 100 & 18.9 & 79296 & 21.4 & -0.64 & 0.525 \\
Separado & 2 & 0.4 & 928 & 0.3 & 0.03 & 0.977
\end{tabular}

*Una significancia menor a 0.05 indicaría una diferencia estadísticamente relevante entre los porcentajes de sentenciados por homicidio y sentenciados en general por delitos dolosos. No se incluyeron los sentenciados con situación conyugal no especificada (NE)

Fuente: Elaboración propia con base en las Estadísticas judiciales en materia penal (INEGI).

Gráfico 5. Homicidio y general (dolosos): proporción de sentenciados con un grado profesional de escolaridad, 1997-2010*

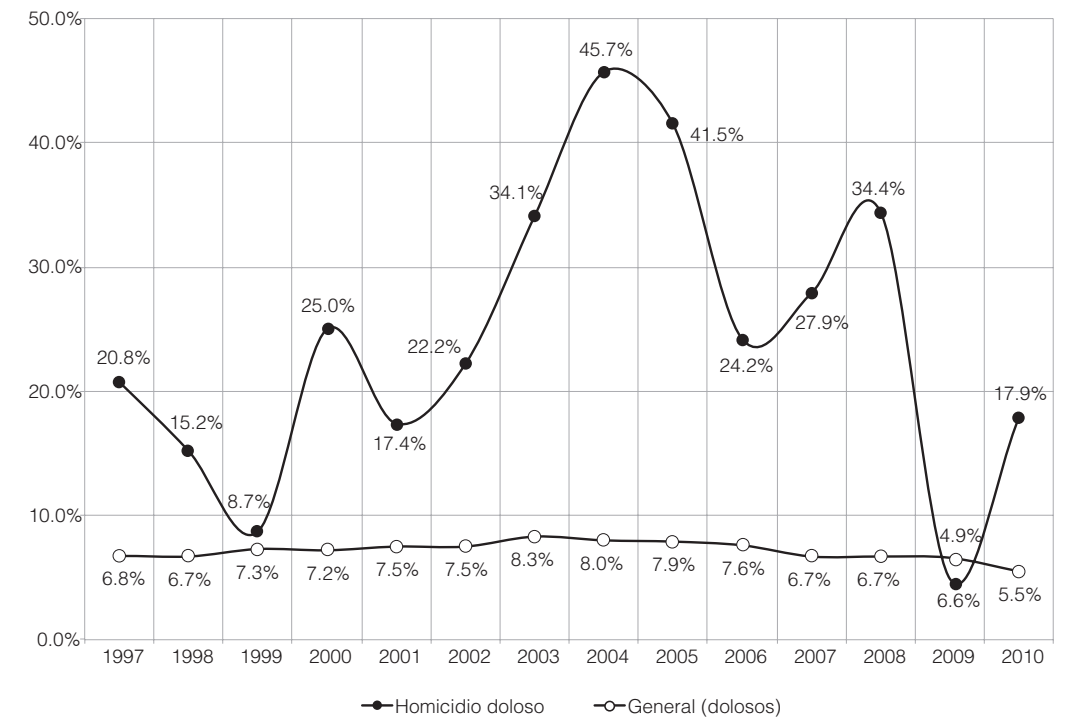

*Un grado profesional es un grado universitario (pregrado y posgrado)

Fuente: Elaboración propia con base en las Estadísticas judiciales en materia penal (INEGI).

En cuanto al nivel de escolaridad, se observa una mayor proporción de grados profesionales entre los sentenciados por homicidio doloso (24.8\%) que en el general $(7.1 \%)$ de los sentenciados. ${ }^{15}$ No se observa, sin embargo,

15 En la mayoría de los años esta diferencia es estadísticamente significativa; no se agrega el cuadro con los resultados de la prueba $\mathrm{Z}$ de diferencias, en este caso por razones de espacio. 
una tendencia clara en el tiempo hacia una mayor o menor proporción de sentenciados con grado profesional entre los homicidas dolosos (gráfico 5). Invariablemente, asimismo, el nivel de escolaridad más frecuente es la primaria $(33.3 \%)$.

Cuadro 9. Homicidio y general (dolosos): escolaridad de los sentenciados, 1997-2010*

\begin{tabular}{|c|c|c|c|c|c|c|c|}
\hline & \multicolumn{7}{|c|}{ Homicidio doloso } \\
\hline & $N$ & $\begin{array}{c}\text { Con escolari- } \\
\text { dad (en \%) }\end{array}$ & $\begin{array}{c}\text { Primaria (en } \\
\%)\end{array}$ & $\begin{array}{l}\text { Secundaria** } \\
\text { (en \%) }\end{array}$ & $\begin{array}{c}\text { Preparatoria** } \\
\text { (en \%) }\end{array}$ & $\begin{array}{l}\text { Profesional } \\
\text { (en \%) }\end{array}$ & Otra (en \%) \\
\hline 1997 & 24 & 100.0 & 45.8 & 29.2 & 4.2 & 20.8 & 0.0 \\
\hline 1998 & 47 & 97.9 & 32.6 & 21.7 & 28.3 & 15.2 & 2.2 \\
\hline 1999 & 28 & 82.1 & 34.8 & 30.4 & 21.7 & 8.7 & 4.3 \\
\hline 2000 & 21 & 95.2 & 60.0 & 10.0 & 5.0 & 25.0 & 0.0 \\
\hline 2001 & 23 & 100.0 & 52.2 & 13.0 & 17.4 & 17.4 & 0.0 \\
\hline 2002 & 37 & 97.3 & 25.0 & 25.0 & 27.8 & 22.2 & 0.0 \\
\hline 2003 & 44 & 93.2 & 39.0 & 17.1 & 7.3 & 34.1 & 2.4 \\
\hline 2004 & 39 & 89.7 & 31.4 & 17.1 & 5.7 & 45.7 & 0.0 \\
\hline 2005 & 41 & 100.0 & 17.1 & 19.5 & 19.5 & 41.5 & 2.4 \\
\hline 2006 & 73 & 84.9 & 38.7 & 17.7 & 17.7 & 24.2 & 1.6 \\
\hline 2007 & 46 & 93.5 & 25.6 & 18.6 & 27.9 & 27.9 & 0.0 \\
\hline 2008 & 32 & 100.0 & 25.0 & 18.8 & 21.9 & 34.4 & 0.0 \\
\hline 2009 & 43 & 95.3 & 29.3 & 36.6 & 24.4 & 4.9 & 4.9 \\
\hline 2010 & 29 & 96.6 & 32.1 & 17.9 & 32.1 & 17.9 & 0.0 \\
\hline \multirow[t]{3}{*}{ Total } & 24 & 100.0 & 45.8 & 29.2 & 4.2 & 20.8 & 0.0 \\
\hline & \multicolumn{7}{|c|}{ General (dolosos) } \\
\hline & $N$ & $\begin{array}{c}\text { Con escolari- } \\
\text { dad (en \%) }\end{array}$ & $\begin{array}{c}\text { Primaria (en } \\
\%)\end{array}$ & $\begin{array}{l}\text { Secundaria** } \\
\text { (en \%) }\end{array}$ & $\begin{array}{c}\text { Preparatoria** } \\
\text { (en \%) }\end{array}$ & $\begin{array}{l}\text { Profesional } \\
\text { (en \%) }\end{array}$ & Otra (en \%) \\
\hline 1997 & 19323 & 90.2 & 51.5 & 29.9 & 11.8 & 6.8 & 0.0 \\
\hline 1998 & 21086 & 90.0 & 50.8 & 29.8 & 12.1 & 6.7 & 0.6 \\
\hline 1999 & 24184 & 89.6 & 48.8 & 30.9 & 12.4 & 7.3 & 0.5 \\
\hline 2000 & 23017 & 90.4 & 48.5 & 30.3 & 13.4 & 7.2 & 0.6 \\
\hline 2001 & 24273 & 89.7 & 47.0 & 31.4 & 13.5 & 7.5 & 0.6 \\
\hline 2002 & 25960 & 91.1 & 45.0 & 32.5 & 14.1 & 7.5 & 0.8 \\
\hline 2003 & 27175 & 91.6 & 44.8 & 31.9 & 14.3 & 8.3 & 0.7 \\
\hline 2004 & 28405 & 92.2 & 43.3 & 33.5 & 14.6 & 8.0 & 0.6 \\
\hline 2005 & 28252 & 92.4 & 42.2 & 34.7 & 14.7 & 7.9 & 0.6 \\
\hline 2006 & 28892 & 92.8 & 41.7 & 35.1 & 14.9 & 7.6 & 0.6 \\
\hline 2007 & 28180 & 93.4 & 41.0 & 36.0 & 15.7 & 6.7 & 0.5 \\
\hline 2008 & 27664 & 93.6 & 39.4 & 36.6 & 16.8 & 6.7 & 0.5 \\
\hline 2009 & 28075 & 94.2 & 37.6 & 37.6 & 18.0 & 6.6 & 0.2 \\
\hline 2010 & 35674 & 94.5 & 36.1 & 39.6 & 18.7 & 5.5 & 0.1 \\
\hline Total & 19323 & 90.2 & 51.5 & 29.9 & 11.8 & 6.8 & 0.0 \\
\hline
\end{tabular}

* Los porcentajes de cada nivel de escolaridad suman $100 \%$ porque se calculan sobre el total de los sentenciados con escolaridad. No se incluyeron los sentenciados con escolaridad no especificada (NE).

** $\mathrm{Y}$ equivalentes.

Fuente: Elaboración propia con base en las Estadísticas judiciales en materia penal (INEGI). 
Pasando a la estructura de empleo, los sentenciados por homicidio doloso en el fuero federal destacan por mostrar con elevada frecuencia las siguientes tres ocupaciones: trabajadores agropecuarios (23.3\%), artesanos y obreros $(15.7 \%)$ y profesionistas (14.9\%). Es llamativo, a diferencia del caso de los procesados, que en pocas ocupaciones se observan diferencias estadísticamente significativas entre sentenciados por homicidio doloso y el general de los sentenciados. Precisamente, las únicas dos ocupaciones diferenciales son dos de las más frecuentes y previamente mencionadas: se ve una menor proporción de artesanos y obreros y una mayor proporción de profesionistas entre los sentenciados por homicidio doloso. En las demás ocupaciones se observan proporciones similares o un perfil de empleo no distinguible entre ambos grupos, lo cual contrasta con la población de procesados, en donde se encontraba también una alta y estadísticamente significativa proporción de ocupados en labores de vigilancia y protección dentro del grupo de homicidas dolosos. ${ }^{16}$

El estado psicofísico es otro correlativo importante del comportamiento criminal. En este caso, entre los sentenciados por homicidio doloso, se observa que la enorme mayoría (86.1\%) cometió tal delito en pleno uso de sus facultades, y que esta amplia proporción no es significativamente diferente al del grupo general o de comparación. Si bien en la población de procesados se encontraba una significativamente mayor proporción de homicidas dolosos en tal categoría de pleno uso de facultades, en este caso, o entre la población de sentenciados, para el mismo periodo 1997-2010, no se detecta tal diferencia.

Respecto del grado de consumación del delito, surge aquí otra diferencia entre la población de sentenciados y procesados por homicidio doloso. Si bien entre los procesados por este delito se encontraban, de manera consistente, entre 1997 y 2010 , mayores frecuencias de tentativa (en proceso penal) que entre los procesados en general o por otros delitos, éste no es el caso para ningún año entre la población de sentenciados. ${ }^{17}$ En todo caso, la vasta mayoría de los sentenciados por homicidio doloso (95.8\%) y en general por los delitos dolosos $(98.9 \%)$ consumaron o ejecutaron en su totalidad su delito.

16 Esta falta de evidencia de una diferencia estadísticamente significativa obedece al reducido número o tamaño de muestra de sentenciados por homicidio doloso dentro de tal ocupación $(\mathrm{n}=35)$; en todo caso, éstos representan más del doble (7.0\%) frente al grupo general de sentenciados (3.0\%) al interior de cada grupo, respectivamente.

17 La explicación estadística se encuentra en los pequeños tamaños de muestra de cada ańo y en el total $(\mathrm{n}=22)$ dentro de los sentenciados por homicidio doloso. En todo caso, no hay evidencia suficiente para argumentar una posible diferencia dentro del periodo analizado. Podría hacerse la prueba inversa, es decir, entre proporciones de sentenciados con delitos consumados, pero en este caso la categoría de interés era la tentativa. 
Cuadro 10. Homicidio y general (dolosos): sentenciados por ocupación, 1997-2010

\begin{tabular}{l|cc|cc|cc|}
\cline { 2 - 6 } & \multicolumn{2}{c|}{ Homicidio doloso } & \multicolumn{2}{c|}{ General (dolosos) } & \multicolumn{2}{c|}{ Prueba de diferencias* } \\
\cline { 2 - 7 } & $N$ & $\%$ & $N$ & $\%$ & $Z$ & Sig. \\
\hline PEA: & 503 & 95.1 & 339867 & 91.3 & 3.95 & 0.000 \\
Artesanos y obreros & 79 & 15.7 & 86604 & 25.5 & -2.39 & 0.017 \\
Ayudantes y similares & 14 & 2.8 & 16308 & 4.8 & -0.46 & 0.647 \\
Comerciantes y dependientes & 44 & 8.7 & 47414 & 14.0 & -1.22 & 0.222 \\
Coords. y supervisores admvos. & 2 & 0.4 & 373 & 0.1 & 0.06 & 0.948 \\
Funcionarios y directivos & 1 & 0.2 & 1678 & 0.5 & -0.07 & 0.947 \\
Inspectores y supervisores & 3 & 0.6 & 1153 & 0.3 & 0.06 & 0.954 \\
Oficinistas & 56 & 11.1 & 32362 & 9.5 & 0.38 & 0.702 \\
Operadores de maquinaria fija & 3 & 0.6 & 2542 & 0.7 & -0.03 & 0.973 \\
Operadores de transporte & 48 & 9.5 & 26715 & 7.9 & 0.40 & 0.692 \\
Profesionistas & 75 & 14.9 & 4284 & 1.3 & 3.32 & 0.001 \\
Protección y vigilancia & 35 & 7.0 & 10162 & 3.0 & 0.92 & 0.357 \\
Técnicos & 11 & 2.2 & 4644 & 1.4 & 0.19 & 0.853 \\
Trabajadores agropecuarios & 117 & 23.3 & 79582 & 23.4 & -0.04 & 0.968 \\
Trabajadores ambulantes & 1 & 0.2 & 6454 & 1.9 & -0.38 & 0.703 \\
Trabajadores de la educación & 5 & 1.0 & 2418 & 0.7 & 0.06 & 0.949 \\
Trabajadores del arte & 4 & 0.8 & 2394 & 0.7 & 0.02 & 0.984 \\
Trabajadores domésticos & 0 & 0.0 & 1703 & 0.5 & $n . a$. & n.a. \\
Trabajadores en serv. públicos & 5 & 1.0 & 13077 & 3.8 & -0.64 & 0.520 \\
No PEA: & 26 & 4.9 & 32504 & 8.7 & -0.90 & 0.369 \\
\hline Una signtian
\end{tabular}

*Una significancia menor a 0.05 indicaría una diferencia estadísticamente relevante entre los porcentajes de sentenciados por homicidio y sentenciados en general por delitos dolosos. No se incluyeron los sentenciados con ocupación no especificada (NE). Se incluyen los resultados para ambas proporciones de la variable dicotómica PEA/No PEA, en donde la significancia es diferente a razón de diversos errores estándar, los cuales son explicados por los tamaños divergentes de muestra. No aplica es n.a.

Fuente: Elaboración propia con base en las Estadísticas judiciales en materia penal (INEGI).

Cuadro 11. Homicidio y general (dolosos): sentenciados por estado psicofísico al momento de cometer el delito, 1997-2010

\begin{tabular}{l|cc|cc|cc}
\cline { 2 - 7 } & \multicolumn{2}{c|}{ Homicidio doloso } & \multicolumn{2}{c|}{ General (dolosos) } & \multicolumn{2}{c}{ Prueba de diferencias* $^{*}$} \\
\cline { 2 - 7 } & $N$ & $\%$ & $N$ & $\%$ & $Z$ & \multicolumn{2}{c}{ Sig. } \\
\hline En pleno uso de & 458 & 86.1 & 325589 & 86.8 & -0.45 & 0.654 \\
sus facultades & 42 & 7.9 & 24334 & 6.5 & 0.34 & 0.736 \\
Ebrio & 5 & 0.9 & 10340 & 2.8 & -0.42 & 0.674 \\
Drogado & 0 & 0.0 & 808 & 0.2 & n.a. & n.a. \\
Otro estado & 27 & 5.1 & 13965 & 3.7 & 0.32 & 0.749 \\
Se ignora & & &
\end{tabular}

*Una significancia menor a 0.05 indicaría una diferencia estadísticamente relevante entre los porcentajes de sentenciados por homicidio y sentenciados en general por delitos dolosos. No se incluyeron los sentenciados con estado psicofísico no especificado (NE). No aplica es n.a.

Fuente: Elaboración propia con base en las Estadísticas judiciales en materia penal (INEGI). 
Cuadro 12. Homicidio y general (dolosos): proporción de sentenciados por tentativa, 1997 a 2010

\begin{tabular}{|c|c|c|c|c|c|c|}
\hline & \multicolumn{2}{|c|}{ Homicidio doloso } & \multicolumn{2}{|c|}{ General (dolosos) } & \multicolumn{2}{|c|}{ Prueba de diferencias* } \\
\hline & $N$ & $\%$ & $N$ & $\%$ & $z$ & Sig. \\
\hline 1997 & 3 & 12.5 & 486 & 2.4 & 0.53 & 0.599 \\
\hline 1998 & 1 & 2.1 & 349 & 1.6 & 0.03 & 0.972 \\
\hline 1999 & 2 & 7.1 & 260 & 1.1 & 0.33 & 0.739 \\
\hline 2000 & 1 & 5.0 & 274 & 1.2 & 0.17 & 0.862 \\
\hline 2001 & 0 & 0.0 & 291 & 1.2 & n.a. & n.a. \\
\hline 2002 & 2 & 5.4 & 222 & 0.9 & 0.28 & 0.777 \\
\hline 2003 & 2 & 4.4 & 167 & 0.6 & 0.26 & 0.794 \\
\hline 2004 & 1 & 2.6 & 199 & 0.7 & 0.12 & 0.906 \\
\hline 2005 & 0 & 0.0 & 221 & 0.8 & n.a. & n.a. \\
\hline 2006 & 1 & 1.4 & 200 & 0.7 & 0.06 & 0.953 \\
\hline 2007 & 0 & 0.0 & 165 & 0.6 & n.a. & n.a. \\
\hline 2008 & 2 & 6.3 & 171 & 0.6 & 0.33 & 0.743 \\
\hline 2009 & 5 & 11.6 & 378 & 1.3 & 0.72 & 0.473 \\
\hline 2010 & 2 & 6.9 & 491 & 1.4 & 0.31 & 0.758 \\
\hline Total & 22 & 4.2 & 3874 & 1.1 & 0.73 & 0.464 \\
\hline
\end{tabular}

*Una significancia menor a 0.05 indicaría una diferencia estadísticamente relevante entre los porcentajes de sentenciados en grado de tentativa por homicidio doloso y sentenciados en general por delitos dolosos. No aplica es n.a.

Fuente: Elaboración propia con base en las Estadísticas judiciales en materia penal (INEGI). El porcentaje se obtuvo sobre el total de procesados, respectivamente.

Gráfico 6. Homicidio y general (dolosos): proporción de procesados por tentativa, 1997-2010

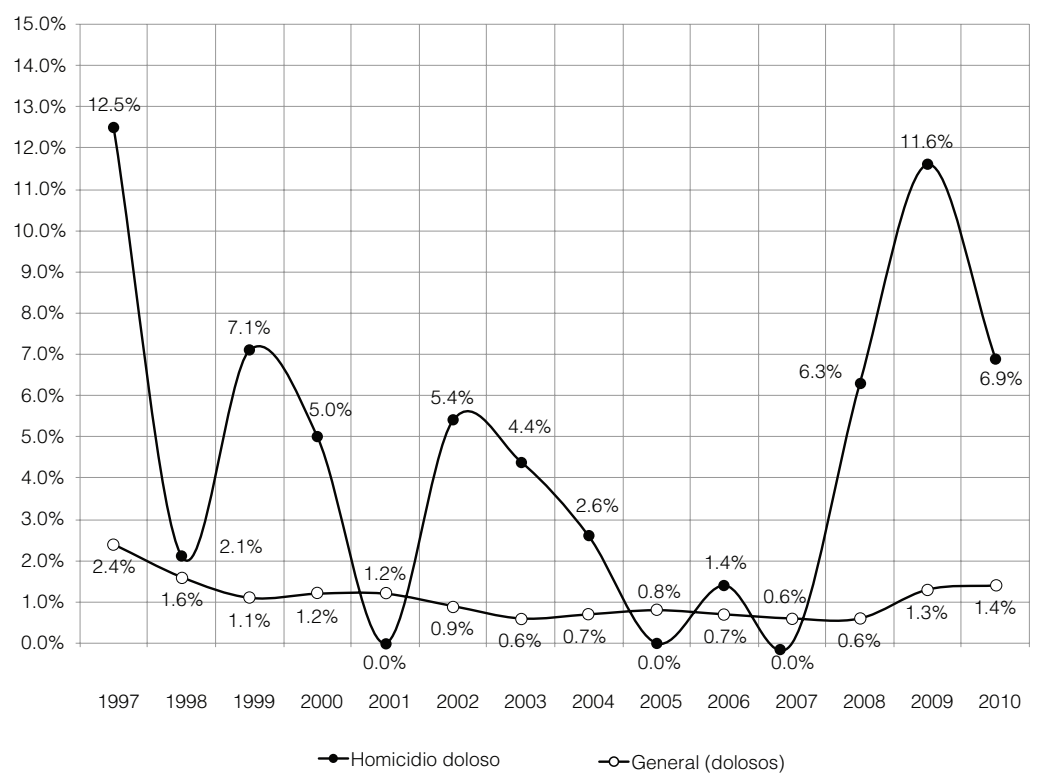

Fuente: Elaboración propia con base en las Estadísticas judiciales en materia penal (INEGI). 
En términos de tendencias temporales (gráfico 6), si bien existen variaciones importantes, se observa que, aunque en las pruebas de significancia no se detectaron diferencias estadísticas, la mayor parte de los años se ha sentenciado, en términos proporcionales, a más homicidas dolosos que en el general de otros delitos dolosos.

En síntesis se encuentra que, dentro del periodo analizado, los sentenciados por homicidio doloso en el fuero federal principalmente residían en el Distrito Federal, Jalisco o el Estado de México, habían cometido el delito en el Distrito Federal, Chiapas o Jalisco (ver cuadros en el anexo B), eran de sexo masculino, tenían entre 30 y 34 o entre 25 y 29 años de edad mayormente, eran de nacionalidad mexicana, casados, con educación primaria o profesional, ocupados en el sector agropecuario, como artesanos u obreros, o bien eran profesionistas, y cometieron el delito en pleno uso de sus facultades, además de que la mayoría de las tentativas fueron consumadas. Estas características muestran al sentenciado por homicidio doloso como parcialmente diferente, al menos en algunos aspectos, al sentenciado por algún delito doloso.

\section{Discusión y recomendaciones para siguientes estudios}

El estudio de la criminalidad en la región se ha limitado a sus aspectos legales. Las discusiones son típicamente normativas y fundamentadas en evidencia anecdótica. Las razones de lo anterior son variadas, pero presumimos que una de las más importantes es la escasez y la novedad de las estadísticas en materia de seguridad y justicia. A diferencia de otras materias de política (por ej., la economía), las estadísticas en esta materia son mucho más recientes y se hallan menos desarrolladas. El enfoque dado en esta investigación sobre las características distintivas de la población de sentenciados por homicidio intencional en México representa el primer intento empírico por sistematizar la información existente y ofrecer las primeras consideraciones al estudio científico del criminal en la realidad de su contexto.

Varios de los hallazgos, aunque iniciales, fueron llamativos. La información que se posee muestra el siguiente perfil general del homicida intencional mexicano en cárceles federales: hombre, entre 25 y 34 años, casado o soltero, ${ }^{18}$ con educación primaria, ocupado en labores manuales como trabajador agropecuario o artesano u obrero, y que cometió su delito en pleno uso de sus facultades. Ésas son sus características generales pero no exclusivas. Lo que lo hace particularmente distinto de los demás son las siguientes características: una proporción

18 En oposición a la unión libre.

Perfiles Latinoamericanos | vol. 23, núm. 45, enero-junio de 2015 | Flacso México Carlos Vilalta | ¿Quiénes son los sentenciados por homicidio intencional en México? | pp. 157-180 
significativamente mayor de casados, con educación universitaria, y ocupados como profesionistas al momento de cometer su delito. Otro hallazgo interesante fue que son un número realmente reducido entre la población penitenciaria federal, son cada vez menos, proporcionalmente, y cerca de un tercio cometió el homicidio fuera del país, principalmente en los Estados Unidos; es decir, que fueron extraditados.

El enfoque en las estadísticas judiciales no es suficiente para delimitar científicamente un perfil delictivo. Si bien las estadísticas judiciales ofrecen información sobre ciertas características básicas y ciertamente con un valor analítico, objetivamente se necesita más información sobre otras características individuales y de contexto familiar y comunitario. Hay vacíos críticos en esta fuente de información. Necesitamos datos estadísticos más detallados para efectos prácticos de política pública, ya para políticas de prevención social del delito, como políticas de reinserción social. Por ejemplo, no sabemos otras cuestiones básicas como cuántos son reincidentes, qué delitos cometieron previo al homicidio intencional, o bien, más detalles en cuanto el evento delictivo. Tampoco sabemos nada de sus víctimas. Es así que, sobre la base de estándares científicos, no podríamos ni probar teóricamente alguna hipótesis ni substanciar empíricamente a alguna decisión de política pública sobre la base de la información presentada en esta nota. Es demasiado general y esta área de política requiere muchas precisiones. En este sentido, los hallazgos también llaman la atención sobre la necesidad de un mayor desarrollo de los sistemas de estadística en seguridad y justicia en México; una preocupación raramente atendida hasta hace poco.

En todo caso, la evidencia que se muestra en este estudio demuestra dos cosas. Primero, que el homicida intencional federal en México es diferente en ciertos aspectos a los sentenciados por otros delitos intencionales. Esto, intuitivamente, conlleva implicaciones de política de prevención del delito y de reinserción social específicas, aunque precisamente el motivo original de este estudio es mostrar que no tenemos bases empíricas para concluir lo anterior. Segundo, que una sistematización básica sí puede realizarse en la región sobre la base de estadísticas judiciales penales que realizan simples conteos de variables demográficas y socioeconómicas.

Los incrementos notables en la violencia homicida en México así como la permanencia de las altas tasas de homicidio en otros países como Brasil, Venezuela y Colombia causan una gran preocupación y no se tiene información para realizar diagnósticos suficientes y fundamentar decisiones de política criminal al respecto. La estadística presentada no debe ser difícil de compilar por las autoridades estadísticas de cada país, porque no implica una reestructuración administrativa de largo alcance ni mucho menos una reforma legal. 
Simplemente se trata de empezar el conteo de eventos. Esta información se puede empezar a compilar y compartir públicamente si las autoridades de la región promueven una cultura estadística. Esperamos que sirva como base para otros sistemas de estadística judicial criminal y otros análisis de perfiles delictivos y estudios correccionales en la región.

\section{Referencias}

Ellis, L., K. Beaver y J. Wright, 2009, Handbook of Crime Correlates, Estados Unidos, Academic Press.

Instituto Nacional de Estadística y Geografía (INEGI), 2012, Estadisticas judiciales en materia penal de los Estados Unidos Mexicanos, México, disponible en <http://goo.gl/1N07Jb>.

Instituto Nacional de Estadística y Geografía (INEGI), 2010, Estadisticas judiciales en materia penal de los Estados Unidos Mexicanos, México.

Instituto Nacional de Estadística y Geografía (INEGI), 2007, Sintesis metodológica de las estadísticas judiciales en materia penal, México.

Leri, F., J. Bruneau, y J. Stewart, 2003, “Understanding Polydrug Use: Review of Heroin and Cocaine Co-Use", Addiction, vol. 98, núm. 1, pp. 7-22.

Makkai, T., y J. Payne, 2003, Drugs and Crime: A Study of Incarcerated Male Offenders, Research and Public Policy, Canberra, Australian Institute of Criminology (Research and Public Policy Series núm. 52), disponible en <http://goo.gl/ZrQNFz>.

Pérez, C. et al., 2012, Primera Encuesta a Población Interna en Centros Federales de Readaptación Social, México, Centro de Investigación y Docencia Económicas (CIDE), disponible en $<$ http://goo.gl/3Ch7cv>.

Real Academia Española (RAE), 2014, Diccionario de la Lengua, disponible en <http:// www.rae.es/>.

Shaw, J. et al., 2006, "The Role of Alcohol and Drugs in Homicides in England and Wales", Addiction, vol. 101, núm. 8, pp. 1117-1124.

Vilalta, C., 2010, "Un examen de la relación entre el consumo de sustancias y la comisión de delitos”, Estudios Sociológicos, vol. 28, núm. 82, pp. 113-134.

Vilalta, C., y G. Fondevila, 2013, Perfiles criminales I: frecuencias y descriptivos, México, Centro de Investigación y Docencia Económicas (CIDE). 


\section{Anexo estadístico}

A. Fórmulas

La fórmula del estadístico $Z$ para calcular la probabilidad de una diferencia entre dos proporciones (grupo 1 y grupo 2) es la siguiente:

$$
Z=\frac{p_{1}-p_{2}}{\sqrt{\frac{p_{1}\left(1-p_{1}\right)}{n_{1}}+\frac{p_{2}\left(1-p_{2}\right)}{n_{2}}}}
$$

Las hipótesis estadísticas se definen de la siguiente manera:

$$
\begin{aligned}
& H o: p_{1}=p_{2} \\
& H a: p_{1} \neq p_{2}
\end{aligned}
$$

Como se puede intuir, la lógica de la prueba radica en calcular a cuántos errores estándar se encuentra la proporción de la muestra 1 de la proporción de la muestra 2 para estimar si provienen de poblaciones diferentes o similares. Si la diferencia es mayor a 1.96 errores estándar, esto sugeriría rechazar Ho con un nivel de significancia o probabilidad de obtener tal resultado por simple azar máxima del 5\% ( $\leq 0.05)$. Se asume que la probabilidad esperada de la diferencia con respecto al error estándar sigue un comportamiento normalmente distribuido. 


\section{B. Otros cuadros de estadísticas}

Cuadro 16. México: sentenciados por homicidio doloso según su lugar de residencia al interior y fuera del país, 1997-2010

\begin{tabular}{|c|c|c|}
\hline & Número & Proporción \\
\hline \multicolumn{3}{|l|}{ Al interior del país } \\
\hline Aguascalientes & 2 & $0.4 \%$ \\
\hline Baja California & 31 & $5.8 \%$ \\
\hline Baja California Sur & 2 & $0.4 \%$ \\
\hline Campeche & 5 & $0.9 \%$ \\
\hline Coahuila de Zaragoza & 8 & $1.5 \%$ \\
\hline Colima & 6 & $1.1 \%$ \\
\hline Chiapas & 34 & $6.4 \%$ \\
\hline Chihuahua & 17 & $3.2 \%$ \\
\hline Distrito Federal & 58 & $10.9 \%$ \\
\hline Durango & 9 & $1.7 \%$ \\
\hline Guanajuato & 8 & $1.5 \%$ \\
\hline Guerrero & 23 & $4.3 \%$ \\
\hline Hidalgo & 12 & $2.3 \%$ \\
\hline Jalisco & 56 & $10.5 \%$ \\
\hline México & 48 & $9.0 \%$ \\
\hline Michoacán de Ocampo & 42 & $7.9 \%$ \\
\hline Morelos & 16 & $3.0 \%$ \\
\hline Nayarit & 14 & $2.6 \%$ \\
\hline Nuevo León & 5 & $0.9 \%$ \\
\hline Oaxaca & 15 & $2.8 \%$ \\
\hline Puebla & 8 & $1.5 \%$ \\
\hline Querétaro & 5 & $0.9 \%$ \\
\hline Quintana Roo & 5 & $0.9 \%$ \\
\hline San Luis Potosí & 3 & $0.6 \%$ \\
\hline Sinaloa & 19 & $3.6 \%$ \\
\hline Sonora & 15 & $2.8 \%$ \\
\hline Tabasco & 4 & $0.8 \%$ \\
\hline Tamaulipas & 13 & $2.4 \%$ \\
\hline Tlaxcala & 7 & $1.3 \%$ \\
\hline Veracruz de Ignacio de la Llave & 23 & $4.3 \%$ \\
\hline Yucatán & 1 & $0.2 \%$ \\
\hline Zacatecas & 8 & $1.5 \%$ \\
\hline \multicolumn{3}{|l|}{ Fuera del país } \\
\hline Estados Unidos de América & 8 & $1.5 \%$ \\
\hline Países latinoamericanos & 1 & $0.2 \%$ \\
\hline Otros países & 0 & $0.0 \%$ \\
\hline Total & 531 & $100 \%$ \\
\hline
\end{tabular}


Cuadro 17. México: sentenciados por homicidio doloso según el lugar de ocurrencia del delito al interior y fuera del país, 1997-2010

\begin{tabular}{|c|c|c|}
\hline & Número & Proporción \\
\hline \multicolumn{3}{|l|}{ Al interior del país } \\
\hline Aguascalientes & 2 & $0.39 \%$ \\
\hline Baja California & 19 & $3.74 \%$ \\
\hline Baja California Sur & 2 & $0.39 \%$ \\
\hline Campeche & 3 & $0.59 \%$ \\
\hline Chiapas & 34 & $6.69 \%$ \\
\hline Chihuahua & 8 & $1.57 \%$ \\
\hline Coahuila de Zaragoza & 8 & $1.57 \%$ \\
\hline Colima & 3 & $0.59 \%$ \\
\hline Distrito Federal & 61 & $12.01 \%$ \\
\hline Durango & 6 & $1.18 \%$ \\
\hline Guanajuato & 2 & $0.39 \%$ \\
\hline Guerrero & 18 & $3.54 \%$ \\
\hline Hidalgo & 18 & $3.54 \%$ \\
\hline Jalisco & 23 & $4.53 \%$ \\
\hline México & 20 & $3.94 \%$ \\
\hline Michoacán de Ocampo & 12 & $2.36 \%$ \\
\hline Morelos & 14 & $2.76 \%$ \\
\hline Nayarit & 5 & $0.98 \%$ \\
\hline Nuevo León & 3 & $0.59 \%$ \\
\hline Oaxaca & 13 & $2.56 \%$ \\
\hline Puebla & 2 & $0.39 \%$ \\
\hline Querétaro & 3 & $0.59 \%$ \\
\hline Quintana Roo & 6 & $1.18 \%$ \\
\hline San Luis Potosí & 3 & $0.59 \%$ \\
\hline Sinaloa & 10 & $1.97 \%$ \\
\hline Sonora & 10 & $1.97 \%$ \\
\hline Tabasco & 2 & $0.39 \%$ \\
\hline Tamaulipas & 7 & $1.38 \%$ \\
\hline Tlaxcala & 7 & $1.38 \%$ \\
\hline Veracruz de Ignacio de la Llave & 22 & $4.33 \%$ \\
\hline Yucatán & 1 & $0.20 \%$ \\
\hline Zacatecas & 2 & $0.39 \%$ \\
\hline \multicolumn{3}{|l|}{ Fuera del país } \\
\hline Estados Unidos de América & 151 & $29.72 \%$ \\
\hline Otros países & 6 & $1.18 \%$ \\
\hline Países latinoamericanos & 2 & $0.39 \%$ \\
\hline Total & 508 & $100 \%$ \\
\hline
\end{tabular}

Recibido el 26 de diciembre de 2012. Aceptado el 22 de septiembre de 2014. 